\title{
Use of Hydrophilic Monomers to Avoid Secondary Particle Nucleation in Miniemulsion Polymerizations of Methyl Methacrylate
}

\author{
Ana Cristina Bomfim Peixoto, Izabella Maria Ferreira Campos, Helen Conceição Ferraz \\ and José Carlos Pinto
}

\author{
Programa de Engenharia Química/COPPE, Universidade Federal do Rio de Janeiro, Cidade Universitária, \\ CP:68502, Rio de Janeiro, 21941-972 RJ, Brazil
}

\begin{abstract}
Miniemulsion polymerization is widely used to produce polymer nanoparticles. In many applications, it is important to ensure the narrow particle size distribution of the final product, which means that secondary micellar and homogeneous nucleation must be avoided during the reaction course. The present study proposes the use of hydrophilic comonomers to inhibit the occurrence of secondary particle nucleation in miniemulsion polymerizations of methyl methacrylate. Acrylic acid, metacrylic acid, 2-hydroxy ethyl methacrylate and methacrylamide were used as hydrophilic comonomers. It was observed that the use of small amounts of hydrophilic comonomers in miniemulsion polymerizations promoted by oil-soluble initiators could prevent secondary particle nucleation and lead to products with more homogeneous particle size distributions.
\end{abstract}

Keywords: Miniemulsion polymerization, nanoparticle, methyl methacrylate, homogeneous nucleation, hydrophilic monomers.

\section{INTRODUCTION}

Poly(methyl methacrylate) (PMMA) is a very versatile polymer that finds applications in many distinct areas [1]. In the biomedical field, for instance, PMMA is used extensively in odontological [2, 3], orthopedic [4-6] and pharmaceutical [7, 8] applications, including the production of nanoparticles for development of drug delivery systems [9-12]. The extensive use of PMMA in the biomedical field is related to many useful properties, such as the excellent mechanical and chemical resistances and the good biocompatibility $[1,8,9]$.

Commercial production of PMMA nanoparticles is usually performed in batch emulsion polymerization processes [13]. However, control of the final polymer properties can be difficult in MMA emulsion polymerizations due to the strong gel effect and high exothermicity of the reaction [14]. Besides, it is very difficult (and most times impossible) to prepare nanoparticles loaded with chemical compounds through classical emulsion polymerizations, as particle nucleation takes place in the micelles [13]. For this reason, PMMA nanoparticles intended for biomedical applications are prepared mainly through miniemulsion polymerizations, as the final particles can be loaded with active components during the preparation of the initial monomer nanodroplets $[15,16]$.

\footnotetext{
*Address correspondence to this author at the Programa de Engenharia Química/COPPE, Universidade Federal do Rio de Janeiro, Cidade Universitária, CP:68502, Rio de Janeiro, 21941-972 RJ, Brazil;

Tel: +552139388337; Fax: +552139388300;

E-mail: pinto@peq.coppe.ufrj.br
}

Nevertheless, the production of PMMA nanoparticles through miniemulsion polymerization still faces some challenges regarding the control of the particle size distribution of the final product. When droplet nucleation prevails, a narrower distribution can usually be obtained [17], which is very convenient for applications related to drug encapsulation [18], as the narrow size distribution can ensure the more uniform performance of the final product. However, both micellar and homogeneous nucleation mechanisms can occur simultaneously in some operation conditions, leading to broad particle size distributions.

Control of the nucleation mechanism in emulsion and miniemulsion polymerizations does not constitute a trivial matter. For instance, if high amounts of emulsifiers are added to the recipe (up to $5 \mathrm{wt} \%$ in respect to monomer), formation of micelles and very small nanoparticles (in the range of 10 to $100 \mathrm{~nm}$ ) cannot be avoided, although control of miniemulsion stability becomes much easier. On the other hand, if the amounts of emulsifiers added to the recipe are not sufficiently high, the stability of the system can be compromised, leading to particle agglomeration. Moreover, lower amounts of emulsifier are needed to produce larger nanoparticles (in the range of 200 to $500 \mathrm{~nm}$ ), as required by many applications 15]. More interesting yet, the decrease of the overall surfactant concentration can lead to formation of larger droplets and smaller interfacial areas, which can contribute with the relative increase of the surfactant concentration in the aqueous phase and formation of micelles; therefore, the simple reduction of the surfactant 
concentration does not guarantee that micellar nucleation can be avoided.

Micellar nucleation occurs when the concentration of surfactant in the reaction medium is above the CMC (critical micelle concentration), leading to formation of micelles, which are preferentially nucleated by freeradicals formed in the aqueous phase because of the very high specific area of the micelles [17]. Micelle formation can be minimized through careful adjustment of the surfactant concentration and homogenization conditions $[15,18]$. Homogeneous nucleation can also take place when polymer chains formed in the aqueous phase reach the maximum solubility limit and precipitate, being more common in surfactant-free emulsion systems, although it can also occur in miniemulsion polymerizations, depending on the monomer solubility, polymer solubility and the initiator concentration in the water phase [17]. Homogeneous nucleation is normally more important when hydrophilic monomers with high solubility in water are added to the formulation, as the mechanism requires the formation of sufficiently high concentrations of polymer in the aqueous phase [18].

As already said, both micellar and homogeneous nucleation mechanisms are not suitable for encapsulation of active compounds, as mass transfer and thermodynamic constraints make difficult the introduction of active compounds into the newly formed polymer particles. Consequently, the occurrence of these undesired secondary nucleation mechanisms during miniemulsion polymerizations results in broad particle size distributions and formation of heterogeneous particle structures [15].

Although it can be difficult to avoid the occurrence of secondary particle nucleation during miniemulsion polymerizations, some strategies can be effective for reduction of secondary rates of particle nucleation. For instance, the increase of the hydrophobe co-stabilizer concentrations can lead to reduction of the monomer concentration in the aqueous phase, while reduction of the initiator concentration can reduce the rate of generation of free-radicals in the water phase [17].

Manipulation of these variables at plant site, however, is undesirable, as the use of co-stabilizers is related to the miniemulsion stability and the rates of initiation affect the rates of polymerization and properties of the final product [16]. On the other hand, the use of oil-soluble initiators can definitely reduce the concentration of free-radicals in the aqueous phase, contributing with the significant decrease of the rates of secondary particle nucleation [19]. However, it is important to say that radical desorption can lead to formation of radicals in the aqueous phase and allow for secondary particle nucleation $[17,20]$.

This work proposes the use of water-soluble monomers, such as acrylic acid (AA), metacrylic acid (MAA), 2-hydroxy ethyl methacrylate (HEMA) and methacrylamide (MAM), for the enhanced control of particle size distributions of the final product in miniemulsion polymerizations of MMA. It is assumed that free-radicals eventually desorbed from emulsified droplets can promote the aqueous polymerization of soluble monomers and prevent secondary nucleation in the reaction system, when the polymer chains are soluble in the aqueous phase and polymer concentrations are not very high. As an additional gain, the use of such polar water-soluble comonomers can eventually introduce reactive groups on the surfaces of the polymer particles, which can be used for posterior functionalization of the surfaces and improvement of biocompatibility, adherence and dispersion stability [21].

Previous works have shown that the rates of homogeneous nucleation can be reduced in miniemulsion polymerizations with the use of watersoluble comonomers, such as acrylic acid (AA) and methacrylic acid (MAA), through manipulation of the characteristic chain length precipitation limit of the water-soluble monomer/polymer system [19]. However, these studies have focused on the modification of the copolymer composition and final copolymer properties of the obtained polymer material, not on the improved control of the final particle size distributions. It is shown in the present work that the use of small amounts of hydrophilic comonomers in miniemulsion polymerizations of MMA promoted by oil-soluble initiators can indeed prevent the secondary particle nucleation and lead to products with more homogeneous particle size distributions, as desired in most biomedical applications of PMMA latexes.

\section{EXPERIMENTAL}

\section{Materials}

Monomers used were methyl methacrylate (MMA, minimum purity of $99.5 \%$, provided by Vetec), 2hydroxyethyl methacrylate (HEMA, minimum purity of $97 \%$, provided by Sigma-Aldrich), acrylic acid (AA, minimum purity of $99 \%$, provided by Vetec), methacrylic acid (MAA, minimum purity of $99 \%$, provided by IQT Industries) and methacrylamide (MAM, 
minimum purity of $99,9 \%$, provided by BASF). Sodium dodecyl sulfate (SDS, minimum purity of $97 \%$, provided by Vetec) was used as stabilizer and $n$-hexadecane (minimum purity of $99 \%$, provided by Vetec) as the hydrophobe co-stabilizer agent. A sodium bicarbonate solution in distillated water $(0.1 \mathrm{wt} \%)$ was used to adjust the $\mathrm{pH}$ of the reaction medium. Potassium persulfate (KPS, minimum purity of $99 \%$, provided by Sigma-Aldrich) and benzoyl peroxide (BPO, minimum purity of $99 \%$, provided by Vetec with 25 wt $\%$ of humidity) were used as initiators. Hydroquinone (minimum purity of $99 \%$, provided by Vetec) was used as inhibitor to halt the reaction after sample withdrawal. All chemicals were used as received, without any further purification.

\section{Miniemulsion Preparation and Polymerization}

The aqueous phase was prepared through addition of the desired amounts of SDS and sodium bicarbonate solution in distillated water. The oil phase was prepared through addition of the desired amounts of $n$ hexadecane and comonomers in MMA (with exception of MAM, which was dissolved in the aqueous phase). The aqueous and oil phases were mixed and homogenized with help of a high speed homogenizer (Turrax, IKA T18) at $8000 \mathrm{rpm}$ for $5 \mathrm{~min}$, producing droplets with approximately $300 \mathrm{~nm}$ in diameter. The sonicator (Branson Digital Sonifier, model 450) was also used to promote the homogenization of the phases. In this case, the mixture was kept in sonication at $80 \mathrm{~W}$ for $5 \mathrm{~min}$. An ice bath was used during the homogenization step in order to avoid the undesired initiator degradation. BPO and KPS were used to initiate the polymerization reactions. BPO was added into the oil phase and KPS was used in the aqueous phase and added directed to the reaction media after reaching the desired temperature.

Table 1 shows the typical initial composition of the prepared miniemulsions. In this case, oil phase corresponds to $35 \mathrm{wt} \%$ of the total emulsion prepared. This oil phase was composed by the monomer MMA, comonomer, when used, and n-hexadecane. Monomer and comonomer represents $25 \mathrm{wt} \%$ and $7 \mathrm{wt} \%$ of the total emulsion, respectively. Other reagents were calculated in respect to the monomer. SDS was used as surfactant agent in the reactions, at a concentration of $1 \mathrm{wt} \%$ in respect to the monomer. This concentration in the aqueous phase corresponds to $2 \mathrm{mM}$, which is below the CMC of SDS (8 mM) [16]. The formulation shown in Table 1 was used as reference for production of PMMA nanoparticles intended for biomedical applications [11, 12].

Polymerization reactions were carried out in a 100 $\mathrm{mL}$ glass reactor (EasyMax102, Mettler Toledo) under controlled temperature and continuous stirring of 500 rpm for $2 \mathrm{~h}$. When the initiator KPS was employed, temperature was set at $70{ }^{\circ} \mathrm{C}$ and the desired amount of KPS was fed into the reaction flask to initiate the reaction. When the initiator $\mathrm{BPO}$ was used, the reaction temperature was kept at $80^{\circ} \mathrm{C}$.

\section{Characterization}

Monomer conversion was monitored through standard gravimetric analyses. Samples were withdrawn and dried at $50{ }^{\circ} \mathrm{C}$ under vacuum until constant weight. The obtained dry polymer material was used to perform thermal (DSC, 8500 Perkin-Elmer) and molecular weight analyses (GPC, VE2001Viscotek, equipped with four Phenomenex columns and a refractometric detector, Viscotek VE3580). Thermal analyses were performed under nitrogen, using heating rates of $10{ }^{\circ} \mathrm{C} / \mathrm{min}^{-1}$. The second heating scan was used for evaluation of thermal properties in order to erase the thermal history of the polymer sample. GPC analyses were performed in tetrahydrofuran $\left(1 \mathrm{mg} \mathrm{mL}^{-1}\right)$ at $40{ }^{\circ} \mathrm{C}$, using PMMA standards with average weight-average molar masses ranging from $5 \times 10^{3}$ to $3 \times 10^{6} \mathrm{Da}$ for calibration. The size distributions of droplets and particles were determined through dynamic light scattering (Nano-ZS, Malvern Instruments) at ambient temperature and using the samples in their original form.

\section{RESULTS AND DISCUSSION}

\section{PMMA Homopolymerization}

Figure 1 shows the evolution of particle size distributions in MMA miniemulsion polymerizations

Table 1: Typical Miniemulsion Formulation

\begin{tabular}{|c|c|c|c|c|c|c|}
\hline \multirow{2}{*}{$\begin{array}{c}\text { Phase } \\
\text { Compound }\end{array}$} & \multicolumn{2}{|c|}{ Aqueous (65 wt\%) } & \multicolumn{3}{|c|}{ Oil (35 wt\%) } & \multirow{2}{*}{$\begin{array}{c}\text { Initiator } \\
\text { BPO/KPS }\end{array}$} \\
\hline & SDS & $\mathrm{NaHCO}_{3}$ & monomer & comonomer & n-hexadecane & \\
\hline wt $\%$ in respect to monomer & 1 & 0.01 & 25 & 7 & 3 & 0.5 \\
\hline
\end{tabular}


performed in accordance with the formulation presented in Table 1, using KPS or BPO as initiators. When KPS was used as initiator (Figure 1a), the occurrence of secondary particle nucleation is evident, with formation of a second peak placed at very small particle diameters after 15 minutes of reaction, even in absence of water-soluble comonomers. The MMA solubility in water is $1.6 \%$ wt at ambient temperature [22] and it is not possible to guarantee that micelles had not been formed inside the reactor, although the initial surfactant concentration was below the CMC [16]. Therefore, secondary particle nucleation can occur even in absence of water-soluble monomers and when the initial surfactant concentration is below the
CMC. Because of the secondary particle nucleation, the final particle size distribution was placed in the region of low particle diameters, around $100 \mathrm{~nm}$.

When BPO was used as initiator (Figure 1b), secondary particle nucleation could not be detected in the initial 30 minutes of reaction (showing that generation of radicals in the water phase can be of fundamental importance for secondary nucleation of polymer particles), but the loss of stability became evident, as peaks positioned at large particle sizes could be observed after $20 \mathrm{~min}$ of reaction. Despite that, it must be clear that coagulum was not obtained at the end of the reaction. As the reaction evolved, the
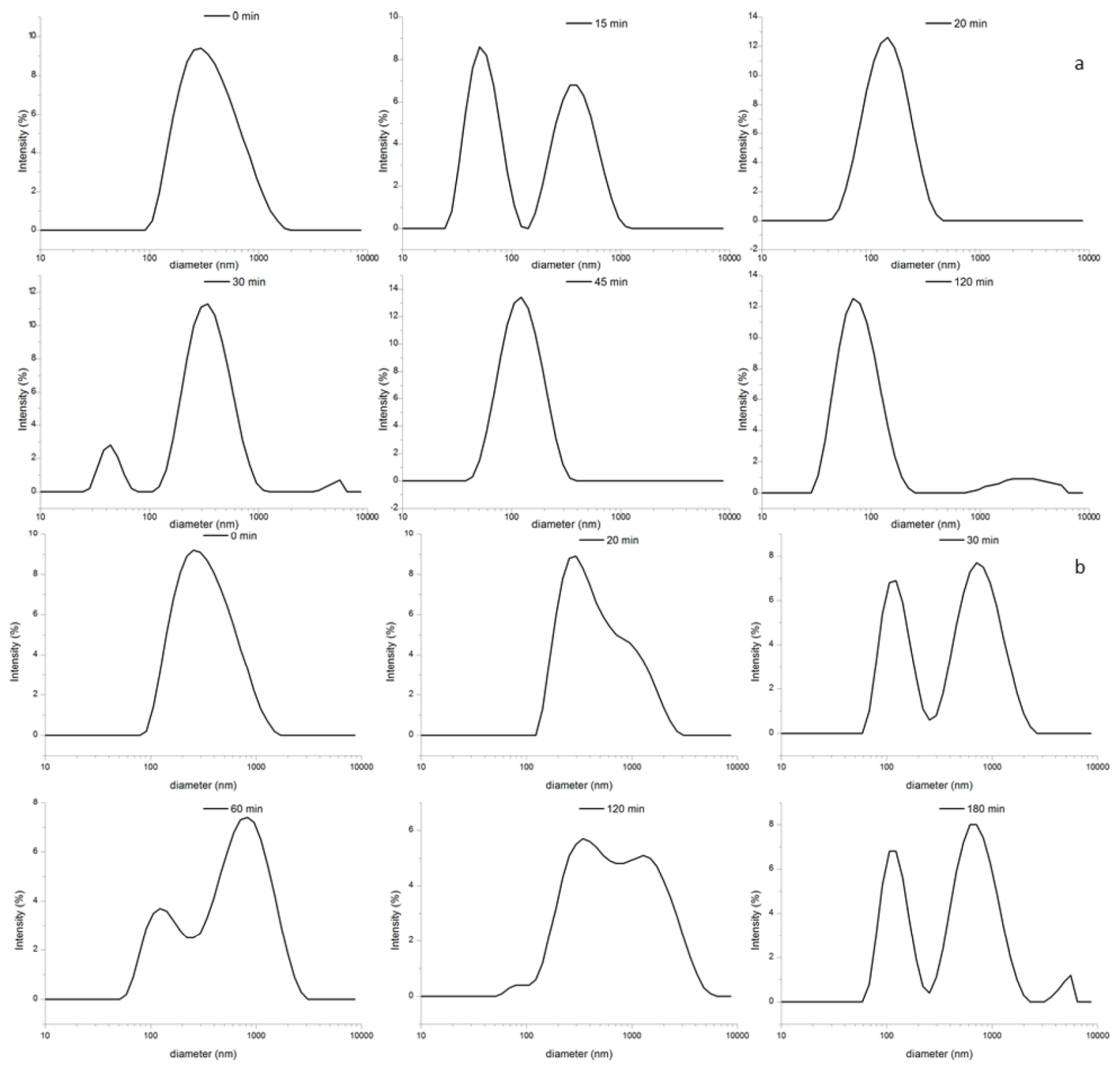

Figure 1: Evolution of particle size distributions in MMA miniemulsion homopolymerizations using a) $\mathrm{KPS}$ at $70{ }^{\circ} \mathrm{C}$ and $\left.\mathbf{b}\right) \mathrm{BPO}$ at $80{ }^{\circ} \mathrm{C}$. 

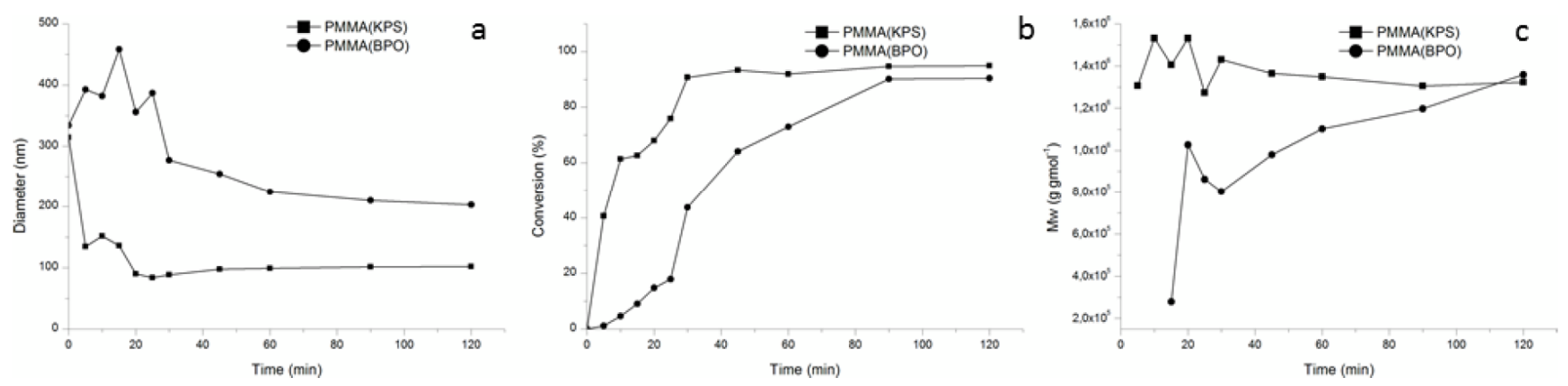

Figure 2: Evolution of a) average particles diameters, b) monomer conversions and c) average molar masses in MMA miniemulsion homopolymerizations performed with KPS at $70^{\circ} \mathrm{C}$ or $\mathrm{BPO}$ at $80^{\circ} \mathrm{C}$.

simultaneous occurrence of particle growth and secondary particle nucleation led to formation of bimodal size distributions, despite the use of similar amounts of surfactant and co-stabilizer, when compared to the reaction performed with KPS. This clearly shows that the rates of polymerization and chemical nature of the initiator can affect the rates of particle nucleation and size distributions and number of polymer particles of the final product. For instance, it is well known that the presence of polar KPS fragments in the polymer chains contribute with the stabilization of the obtained polymer particles [19].

Figure 2 shows the dynamic trajectories of average particle sizes and complements the analysis of the size distributions shown in Figure 1. When BPO was added to the reacting mixture, average sizes increased during the initial 30 min of reaction. As shown in Figure 1, this was related to the fast increase of particle sizes in presence of initiator, before the occurrence of particle nucleation. On the other hand, when KPS was used, the occurrence of a second peak at the region of small diameters due to particle nucleation promoted the initial decrease of the average sizes.

As shown in Figure 2, the dynamic trajectories of monomer conversions and average molar masses of the obtained polymer were very different when KPS and BPO were used as initiators. When KPS was used, reaction rates and molar masses were higher than observed with BPO. First, this is related to the faster rates of initiator decomposition of KPS, when compared to BPO. Second, according to Antonietti [17], when an oil-soluble initiator is used, the radical fragments formed by the initiator thermal decomposition tend to stay inside the droplets, increasing the local concentration of active radicals and enhancing the rates of radical termination. Both effects contribute with the decrease of reaction rates and average molar masses of the polymer product.
Consequently, the slower reaction dynamics also increases the probability for particle agglomeration, especially during intermediate conversion levels. According to Figure 2, monomer conversion was close to $20 \%$ after 20 minutes of reaction when BPO was used as the initiator, while it was close to $80 \%$ when KPS was used as the initiator. Despite the distinct dynamic trajectories, the final monomer conversions, final average molar masses and molecular weight distributions of the final polymer, shown in Figure 3, were somewhat similar in both cases.

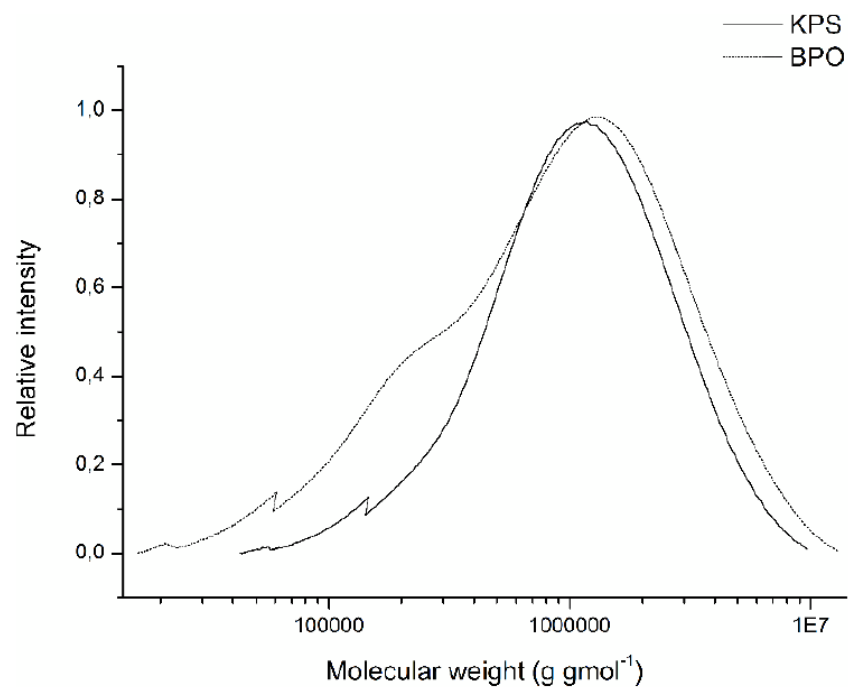

Figure 3: Molecular weight distributions of the final PMMA product for reactions performed with KPS and BPO as initiators.

Figure 4 shows the dynamic evolution of droplet size distributions when no initiator was added to the initial MMA miniemulsion. As shown in Figure 4, although continuous reduction of fractions of very small droplets could be observed, size distributions did not change significantly and the miniemulsion was kept essentially stable for at least $120 \mathrm{~min}$. As a consequence, one can definitely assume that differences observed in the particle size distributions 


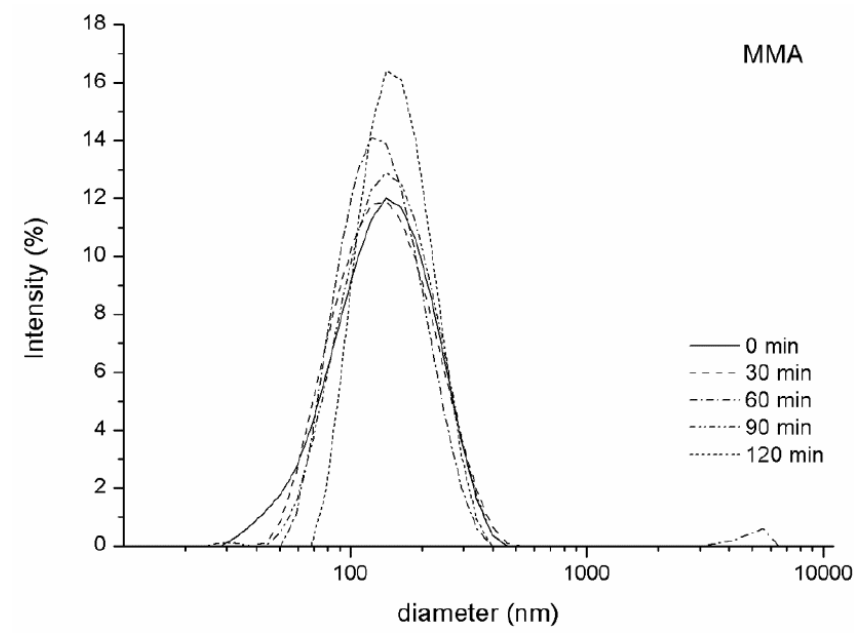

Figure 4: Evolution of droplet size distributions of MMA miniemulsions prepared with the sonicator.

when BPO or KPS were added to the reacting mixture, as shown in Figure $\mathbf{1}$ and Figure $\mathbf{2 a}$, were due to the kinetic behavior of the reaction and not related to the miniemulsion stability. Furthermore, it is important to emphasize that the evolution of average values can be misleading for correct interpretation of the reaction trajectory, as some of the reported size distributions presented multimodal characteristics.
The method used to prepare the initial dispersion of oil droplets in water can exert significant influence on the evolution of particle size distributions, as shown in Figure 5 and discussed in the literature [18]. Emulsions prepared through sonication normally present much narrower size distributions. This can significantly affect the final size distributions of products prepared with oilsoluble initiators, as the larger particles facilitate the particle agglomeration and production of multimodal particle size distributions. Besides, the presence of smaller particles (and larger specific interfacial areas) makes droplet nucleation more probable, also allowing for production of more uniform size distributions at the end of the reaction. The stabilizing effect of KPS fragments become evident in Figure $\mathbf{5 a}$, as the final size distributions in both cases are placed in the region of low particle diameters, as also discussed previously.

\section{Copolymerization with Water-Soluble Monomers}

Table 2 summarizes the results obtained after characterization of the prepared copolymers. The Tg's of the final PMMA homopolymers were similar, regardless the initiator used to perform the miniemulsion polymerization $\left(117.5{ }^{\circ} \mathrm{C}\right.$ for $\mathrm{KPS}$ and $116.7{ }^{\circ} \mathrm{C}$ for BPO). This result was expected, as the

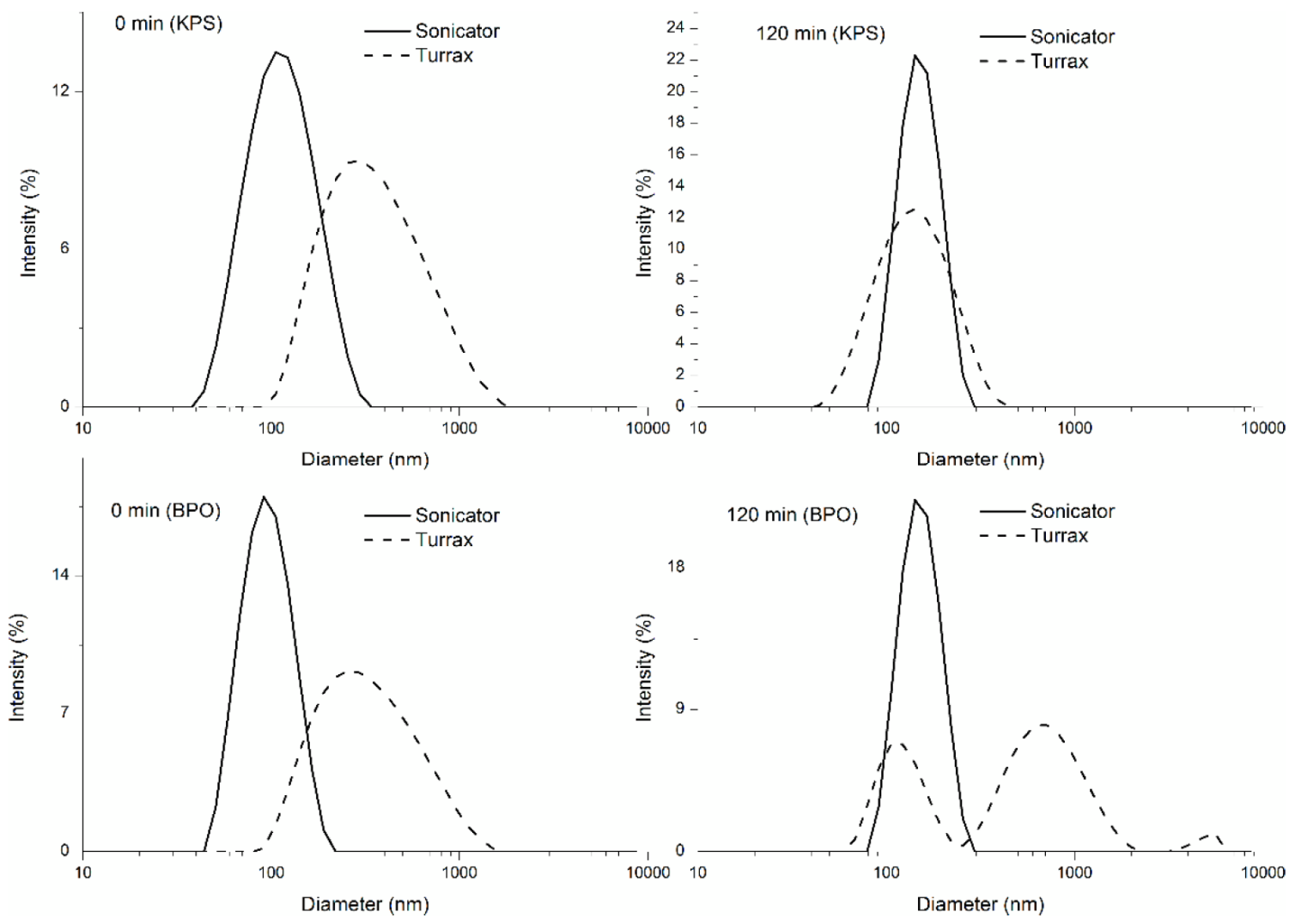

Figure 5: Evolution of particle size distributions in MMA miniemulsion homopolymerizations using different dispersion devices and KPS at $70^{\circ} \mathrm{C}$ or BPO at $80^{\circ} \mathrm{C}$. 
Table 2: Results of Copolymer Characterization

\begin{tabular}{|c|c|c|c|c|c|c|c|c|c|c|c|c|c|}
\hline \multirow{3}{*}{ Polymer } & \multicolumn{4}{|c|}{ Average diameter (nm) } & \multicolumn{3}{|c|}{ Glass transition $-\mathrm{Tg}\left({ }^{\circ} \mathrm{C}\right)$} & \multicolumn{4}{|c|}{ Molecular weight } & \multirow{2}{*}{\multicolumn{2}{|c|}{$\begin{array}{c}\text { Conversion } \\
(\%)\end{array}$}} \\
\hline & \multicolumn{2}{|c|}{ Initial } & \multicolumn{2}{|c|}{ Final } & \multirow[t]{2}{*}{$\begin{array}{c}\text { Comonomer } \\
\text { homopolymer } \\
\mathrm{Tg}^{22}\end{array}$} & \multicolumn{2}{|c|}{$\begin{array}{l}\text { Copolymer Tg } \\
\text { measured by } \\
\text { DSC }\end{array}$} & \multicolumn{2}{|c|}{$\begin{array}{c}\mathrm{Mw} \\
\left(\mathrm{g} \mathrm{gmol}^{-1}\right) \times \\
10^{-5}\end{array}$} & \multicolumn{2}{|c|}{ Polydispersity } & & \\
\hline & KPS & BPO & KPS & BPO & & KPS & BPO & KPS & BPO & KPS & BPO & KPS & BPO \\
\hline PMMA & 314 & 334 & 102 & 203 & - & 117.6 & 116.7 & 13 & 14 & 2.0 & 3.8 & 94.8 & 90.4 \\
\hline P(MMA-Co-HEMA) & 252 & 256 & 94 & 375 & 85 & 114.1 & 115.7 & 38 & 11 & 11.9 & 7.3 & 99 & 85.5 \\
\hline P(MMA-co-MAA) & 243 & 245 & 172 & 569 & 228 & 130.2 & 125.6 & 10 & 7.9 & 6.7 & 4.0 & 93.3 & 37.7 \\
\hline P(MMA-co-AA) & 250 & 237 & 115 & 510 & $75-106$ & 108.7 & 109.2 & 13 & 6.3 & 2.7 & 3.2 & 97.9 & 87.1 \\
\hline P(MMA-co-MAM) & - & 308 & - & 237 & 38.5 & - & 108.8 & - & 7.3 & - & 3.2 & - & 85.6 \\
\hline
\end{tabular}

final molecular weight distributions of PMMA samples were also similar in both cases. The existence of single thermal transitions for all the obtained copolymers suggests the occurrence of copolymerization, instead of mixing of distinct homopolymer chains.

Based on the observed $\mathrm{Tg}$ values, the copolymer composition was estimated with the well-known Fox equation [23] as shown in Table 3. Despite of the accuracy of the results presented in Table 3 , given the limitations of the Fox equation, it is important to report the significant incorporation of comonomers in all cases and the higher comonomer incorporations obtained with KPS, which is in perfect agreement with the high solubility of all monomers in the water phase and the expected higher concentration of radicals in the aqueous phase when KPS is the initiator.

Table 3: Final Copolymer Composition Calculated with Tg Values and Fox Equation [23]

\begin{tabular}{|c|c|c|}
\hline \multirow{2}{*}{ Polymer } & \multicolumn{2}{|c|}{ Composition (mol\%) } \\
\cline { 2 - 3 } & BPO & KPS \\
\hline \hline P(MMA-co-HEMA) & 2.32 & 7.28 \\
\hline P(MMA-co-MAA) & 14.52 & 20.48 \\
\hline P(MMA-co-AA) & 12.35 & 13.96 \\
\hline P(MMA-co-MAM) & 3.57 & $-^{a}$ \\
\hline
\end{tabular}

${ }^{a}$ Value was not detected.

When KPS was used as initiator, the particle size distributions were shifted towards smaller diameters in all cases, due to secondary particle nucleation, as shown in Figure 6. However, as described in the literature, the addition of $A A$ and MAA into the reaction system retards the occurrence of secondary nucleation, due to stabilization of radical and dead polymer chains in the aqueous phase. Nevertheless, when secondary particle nucleation is started after $30 \mathrm{~min}$ of reaction, significant shifting of particle size distributions can be observed towards the region of very small polymer particles. The use of MAM led to massive coagulation of polymer particles, due to the increase of viscosity caused by the accumulation of MAM homopolymer in the aqueous phase at the analyzed conditions, preventing the establishment of the miniemulsion polymerization. The use of HEMA did not affect the evolution of size distributions significantly, when compared to the MMA homopolymerization.

When BPO was used as initiator, the effects of comonomer addition on the evolution of the particle size distributions were not uniform, depending on the comonomer used. It is important to emphasize here that MMA miniemulsion polymerizations prepared with the sonicator also led to occurrence of secondary particle nucleation phenomena, as shown in Figure 7. This is probably related to the smaller particle diameters in this case, which can lead to more significant desorption of radicals from emulsified droplets. However, when AA, MAA and HEMA were used, the secondary particle nucleation was almost completely suppressed, as shown in Figure $\mathbf{6}$, constituting a very beneficial effect for the MMA polymerization. Besides, the use of BPO also allowed for successful miniemulsion polymerizations in presence of MAM, as aqueous polymerization of MAM could be reduced significantly due to radical initiation inside the droplets.

In fact, particle agglomeration was also reduced very significantly, although the average particle diameters increased during the reaction course. The reduction of rates of secondary nucleation can be related to the stabilization of radical and dead polymer chains in the aqueous phase, as discussed previously. However, as radical concentrations are expected to be much lower when oil-soluble initiators are used, limiting 


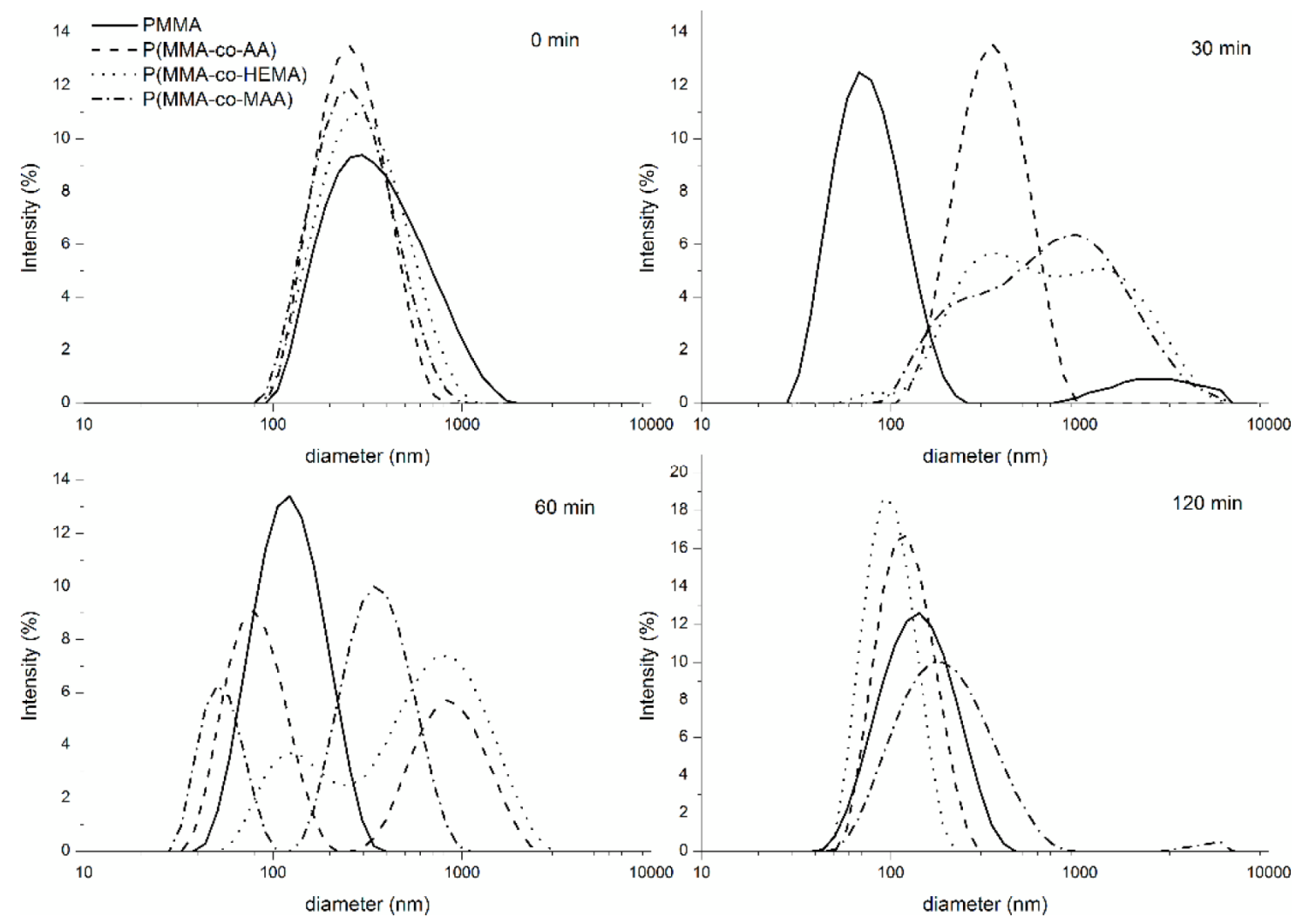

Figure 6: Evolution of particle size distributions in MMA miniemulsion copolymerizations using KPS at $70{ }^{\circ} \mathrm{C}$ and Turrax for homogenization.
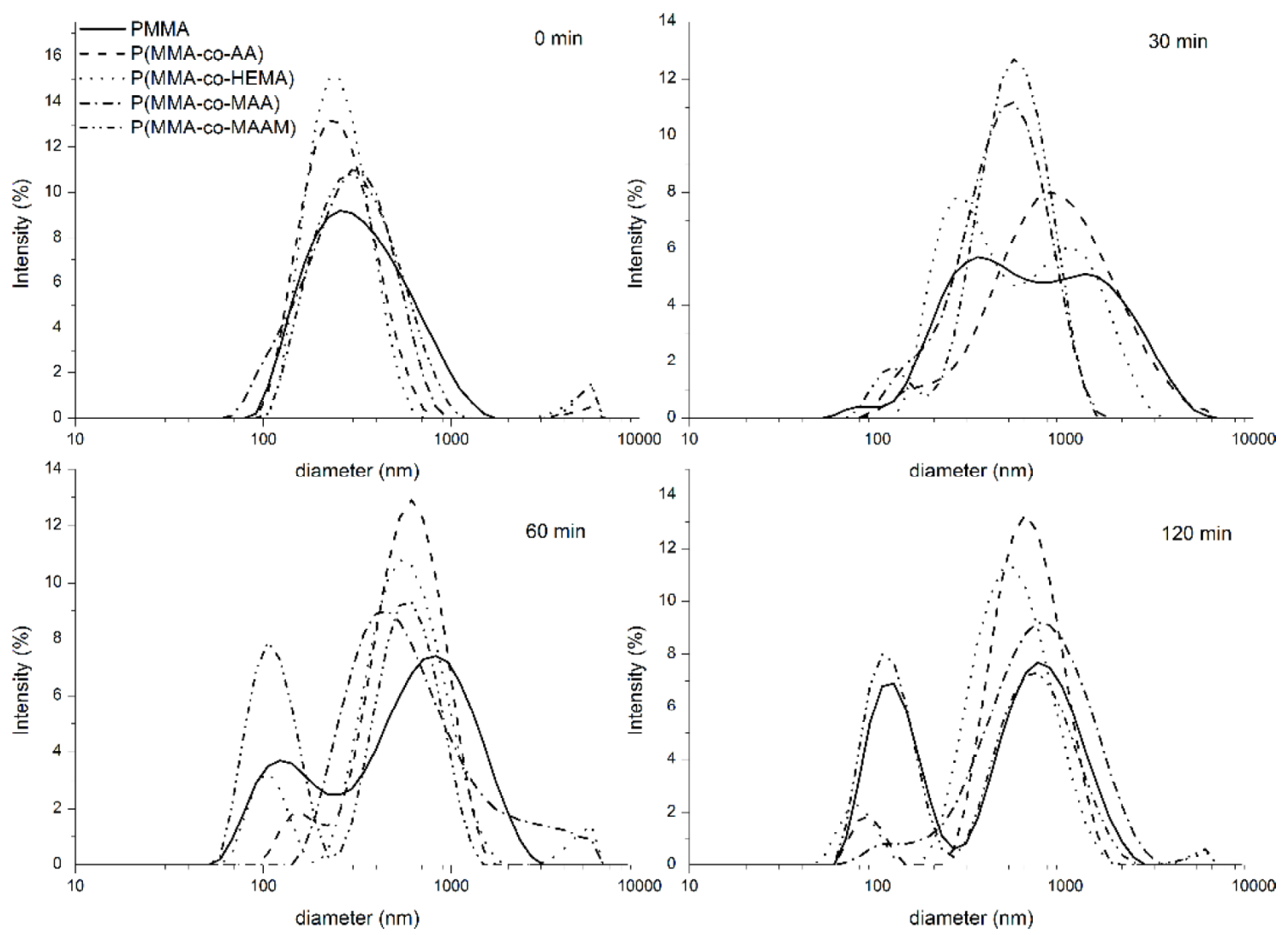

Figure 7: Evolution of particle size distributions in MMA miniemulsion copolymerizations using $\mathrm{BPO}$ at $80^{\circ} \mathrm{C}$ and sonicator for homogenization. 
concentration values for precipitation are not reached on the reaction course. The reduction of rates of agglomeration can be related both to the dispersion device (as shown previously, sonication leads to more uniform size distributions), faster reaction rates and to the formation of polar copolymer chains, through incorporation of comonomer. Both effects tend to enhance the stabilization of the particle surfaces, allowing for production of more stable polymer latexes. The observed increase of particles diameters in the presence of polar comonomers can also indicate the formation of the so-called polymer brushes on particle surfaces. Polymer brushes can be formed due to the accumulation of hydrophilic copolymer blocks around the particle surface [24], which can bring particles together and increase their effective sizes through entanglement of polymer chains. The use of MAM did not cause any significant change of particle size distributions, when compared to MMA homopolymerizations, which probably indicates that the occurrence of polymerization reactions in the water phase is not sufficient to explain the evolution of particle sizes in the copolymerization systems.

Regarding the evolution of droplet size distributions of the initial miniemulsions when water-soluble monomers were added to the reacting mixture in absence of initiator, it can be seen in Figure 8 that size distributions remained essentially stable during the analyzed time, although some changes of the size distributions could be observed during the initial 120 minutes of rest. Particularly, when AA was added to the reacting mixture, a continuous and slow drift of the size distributions could be observed towards the direction of larger droplet sizes. Despite that, no phase separation could be observed. Besides, Figure 8 shows very clearly that the observed modification of the particle size distributions in presence of initiators could not be attributed to the emulsion stability and were certainly related to the kinetic characteristics of the reacting system. Besides, as already said, it is important to emphasize that the evolution of average values can be misleading for correct interpretation of the reaction trajectories, as some of the reported size distributions presented multimodal characteristics.

Figure 9 shows the evolution of average particle diameters, monomer conversions and average molar masses in MMA miniemulsion copolymerizations performed with $\mathrm{KPS}$ and $\mathrm{BPO}$ at $80{ }^{\circ} \mathrm{C}$. According to Figure 7 , significant modifications of the reaction trajectories occurred when comonomers were fed into
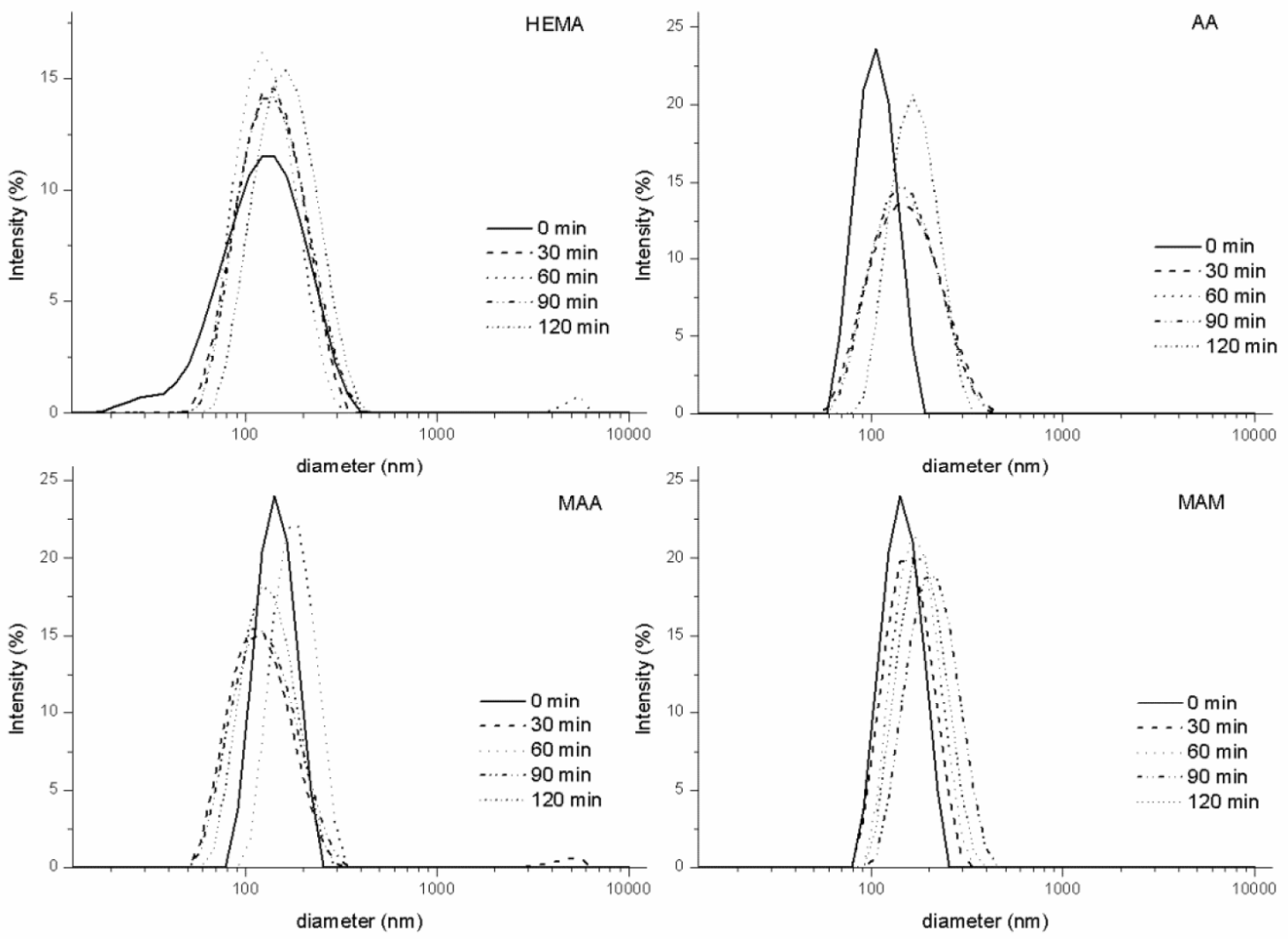

Figure 8: Evolution of droplet size distributions with the water-soluble monomers using sonicator for homogenization. 

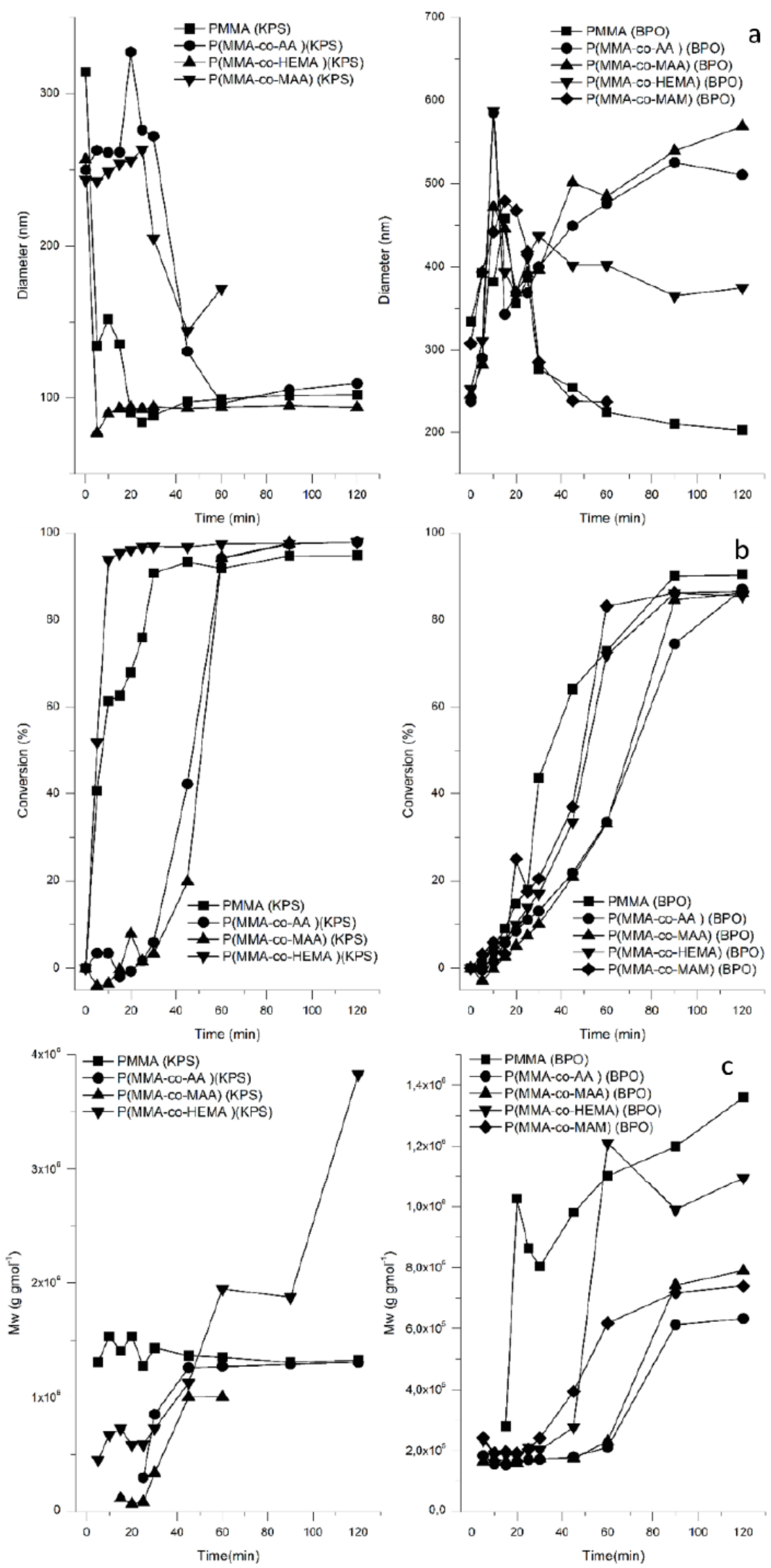

Figure 9: Evolution of a) average particles diameters, b) monomer conversions and c) average molar masses in MMA miniemulsion copolymerizations performed with KPS at $70^{\circ} \mathrm{C}$ or BPO at $80^{\circ} \mathrm{C}$. 
the reaction vessel, when either KPS or BPO were used as initiators. In general, as observed previously, the use of BPO led to lower reaction rates and average molar masses than observed when KPS was used. The induction period observed when $A A$ and MAA were used as comonomers and KPS was used as the initiator is certainly related to reaction in the water phase, which prevents the nucleation of the monomer droplets. The decrease of weight average molar masses in presence of the comonomer is due to their reactivity ratios, which is larger than one for the analyzed copolymerization systems [22].

Materials with very high average molar masses could be obtained when HEMA was used as comonomer, probably because of chain crosslinking, as described in the literature for other similar systems [25].

The addition of MAM to the reaction system did not seem to exert any significant impact on the reaction course, when BPO was used as initiator. This was probably related to the very high solubility of MAM in water (> $10 \% \mathrm{wt}$ ) [22], which reduces the interaction between the reactions that occur simultaneously in both phases, although at much lower rates in the aqueous phase, when compared to the case where KPS is used as initiator. This can be confirmed qualitatively in Table $\mathbf{3}$, which confirms the low incorporation of MAM in the final polymer product.

\section{CONCLUSIONS}

Miniemulsion polymerizations of methyl methacrylate (MMA) were performed in presence of different hydrophilic comonomers, including acrylic acid (AA), metacrylic acid (MAA), 2-hydroxy ethyl methacrylate (HEMA) and methacrylamide (MAM), using initiators soluble in water and in oil. It was observed that the use of hydrophilic comonomers in miniemulsion polymerizations promoted by oil-soluble initiators can prevent the secondary particle nucleation and lead to products with more homogeneous particle size distributions, constituting a very beneficial effect for some biomedical PMMA applications. Particularly, the use of $A A$ and MAA in the feed completely suppressed the occurrence of secondary particle nucleation, while the use of HEMA and MAAM were not very effective, as HEMA presents low solubility and MAM presents too high solubility in water. The reduction of rates of secondary nucleation can be related to the stabilization of radical and dead polymer chains in the aqueous phase. Given the much higher concentration of free-radicals in the aqueous phase when the initiator is soluble in water, similar effects cannot be observed with water-soluble initiators and massive secondary particle nucleation cannot be avoided during the polymerization. Therefore, the combination of a water-soluble comonomer with an oilsoluble initiator can allow for improved control of the nucleation mechanism in MMA miniemulsion polymerizations.

\section{ACKNOWLEDGEMENTS}

Authors would like to thank the Conselho Nacional de Desenvolvimento Científico e Tecnológico (CNPq) for the financial support and scholarships.

\section{REFERENCES}

[1] Oréfice RL. Materiais Poliméricos: Ciência e Aplicação como Biomateriais. In: Oréfice $\mathrm{RL}$, Pereira $\mathrm{M}$, Mansur $\mathrm{H}$ Biomateriais: Fundamentos e Aplicações. Cultura Médica, Rio de Janeiro 2012.

[2] Zheng J, Wang L, Hu Y, Yao K. J Appl Polym Sci 2012; 123 : 2406-13. http://dx.doi.org/10.1002/app.34757

[3] Zuber M, Tabasum S, Jamil T, Shahid M, Hussain R, Feras KS, Bhatti KP. J Appl Polym Sci 2014; 131: 1-9. http://dx.doi.org/10.1002/app.39806

[4] Lemos L, Nele M, Melo P, Pinto JC. Macrol Symp 2006; 243: 13-23.

[5] Santos Jr JG, Pita VJ, Melo P, Nele M, Pinto JC. Braz J Chem Eng 2011; 28: 229-41. http://dx.doi.org/10.1590/S0104-66322011000200007

[6] Bhat KA, Prakash $\mathrm{P}$, Manoharan N, Lakshmibai A, Sangeetha D. J Appl Polym Sci 2013; 127: 2764-75. http://dx.doi.org/10.1002/app.37581

[7] Peixoto LS, Cordeiro FB, Melo P, Nele M, Pinto JC. Macro Symp 2011; 299: 132-8.

[8] Feuser PE, Gaspar PC, Ricci Jr E, da Silva MCS, Nele M, Sayer C, de Araújo PH. Macrol Symp 2014; 343: 65-9.

[9] Mendes NA, Hubber I, Siqueira M, Barbosa G, Moreira DL, Holandino C, Pinto JC, Nele, M. Macrol Symp 2012; 319: 3440.

[10] Oliveira MA, Melo P, Nele M, Pinto JC. Macromol React Eng 2012; 6: 280-92. http://dx.doi.org/10.1002/mren.201100083

[11] Lorca B, Bessa E, Nele M, Santos EP, Pinto JC. Macro Symp 2012; 319: 246-50.

[12] Fonseca LB, Nele M, Volpato NM, Seiceira RC, Pinto JC Macromol React Eng 2013; 7: 54-63. http://dx.doi.org/10.1002/mren.201200036

[13] Chern CS. Prog Polym Sci 2006; 31: 443-86. http://dx.doi.org/10.1016/j.progpolymsci.2006.02.001

[14] O'Neil GA, Wisnudel MB, Torkelson JM. Macrolecules 1996; 22: 7477-90.

http://dx.doi.org/10.1021/ma9606263

[15] Weiss C, Landfester K. Adv Polym Sci 2010; 233: 185-236. http://dx.doi.org/10.1007/12_2010_61

[16] Hecht LL, Wagner C, Landfester K, Schuchmann HP. Langmuir 2011; 27: 2279-85.

http://dx.doi.org/10.1021/la104480s 
[17] Antonietti M, Landfester K. Prog Polym Sci 2002; 27: 1283346.

http://dx.doi.org/10.1016/S0079-6700(01)00051-X

[18] Asua JM. Prog Polym Sci 2002; 27: 689-757. http://dx.doi.org/10.1016/S0079-6700(02)00010-2

[19] Chern CS, Sheu JC. Polymer 2001; 42: 2349-57. http://dx.doi.org/10.1016/S0032-3861(00)00608-X

[20] Capek I. Adv Colloid Interface Sci 2010; 156: 35-61. http://dx.doi.org/10.1016/j.cis.2010.02.006
[21] Pichot C. Curr Opin Colloid Interface Sci 2004; 9: 213-21. http://dx.doi.org/10.1016/j.cocis.2004.07.001

[22] Brandrup J, Immergut EH, Grulke EA. Polymer Handbook. John Wiley \& Sons 2005.

[23] Fox TG. Bull Am Phys Soc 1956; 1: 123.

[24] Holzapfel V, Musyanovch A, Landfester K, Lorenz MR, Mailander V. Macromol Chem Phys 2005; 206: 2440-49. http://dx.doi.org/10.1002/macp.200500372

[25] Qie L. PhD. Thesis. University of Ottawa, Canada 2011.

Received on 25-05-2016

DOI: http://dx.doi.org/10.6000/1929-5995.2016.05.02.2

(C) 2016 Peixoto et al.; Licensee Lifescience Global.

This is an open access article licensed under the terms of the Creative Commons Attribution Non-Commercial License (http://creativecommons.org/licenses/by-nc/3.0/) which permits unrestricted, non-commercial use, distribution and reproduction in any medium, provided the work is properly cited. 